\title{
Galactic Metallicity Distribution from Open Clusters
}

\author{
L. Chen, J.L. Hou, J.J. Wang \\ Shanghai Astronomical Observatory, CAS, Shanghai 200030,China
}

\begin{abstract}
We have compiled two new Galactic open cluster catalogues. The first one has 119 objects with age, distance and metallicity data, while the second one has 144 objects with both absolute proper motion and radial velocity data, of which 45 clusters also with metallicity data available. An iron radial gradient of about $-0.063 \pm 0.008$ dex $\mathrm{kpc}^{-1}$ is obtained from our sample. By dividing clusters into age groups, we show that iron gradient was steeper in the past. A disk age-metallicity relation could very probably exist based on the current sample.
\end{abstract}

\section{Introduction}

Open clusters (OCs) have long been used to trace the structure and evolution of the Galactic disk. Since (OCs) could be relatively accurately dated and can be seen from large distance, their $[\mathrm{Fe} / \mathrm{H}]$ values serve an excellent tracer to the abundance gradient along the Galactic disk as well as many other important disk properties, such as abundance gradient evolution, disk age and so on.

In this paper, we give some statistical results of the open cluster based on our up-to-date sample, mainly relating to the Galactic abundance gradient and age-metallicity relation. Details can be found in Chen, Hou \& Wang (2003).

\section{The catalogues}

We have compiled two new catalogues of the Galactic open clusters. The first one (CAT 1) lists 119 clusters parameters for distance, age and metallicity. The age, distance and reddening information and most iron abundance data are from Dias et al. (2002). Thus far CAT 1 provides a most complete open cluster sample concerning the iron abundance, distance and age parameters together. This sample could provide statistically more significant information concerning the radial iron gradient as well as its evolution, etc. In the second catalogue (CAT 2), we have listed observed kinematical data from literature for 144 clusters, with both radial velocity and mean proper motion available. The mean radial velocity data are mostly from a compilation in WEBDA database. The absolute proper motion of clusters, based on the Hipparcos system, are from Baumgardt et al.(2000) and Dias et al.(2001). In fact, the above observed kinematic information constitute a sub-catalogue of that of Dias et al. (2002). But in CAT 2 we have further calculated the three dimensional velocity of open clusters by combing with radial velocity and mean absolute proper motion data. In ad- 
dition, for each cluster, age and iron abundance data are also listed whenever available. Two catalogues are available online.

\section{Some statistical results}

\subsection{The abundance gradients}

The first radial metallicity gradient using open clusters was given by Janes (1979), with derived gradient of $-0.05 \mathrm{dex} \mathrm{kpc}^{-1}$. Since then a lot of work has been done in the field.

The existence of radial iron abundance gradients is also confirmed by our upto-date sample. By least-square fitting, we derived an radial abundance gradient

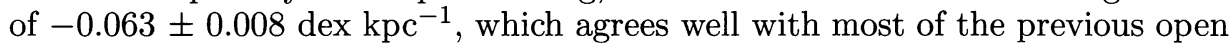
cluster results.

\subsection{Gradient evolution}

We have calculated gradients for two sub-samples with cluster age $<0.8 \mathrm{Gyr}$ (80 clusters) and $\geq 0.8 \mathrm{Gyr}$ (38 clusters), respectively. The fitting results are $-0.024 \pm 0.012 \mathrm{dex} \mathrm{kpc}^{-1}$ for younger clusters, $-0.075 \pm 0.013 \mathrm{dex} \mathrm{kpc}^{-1}$ for older ones. If we take the mean age for the youngest and oldest clusters as 0.00 Gyr and 6.00 Gyr in our sample, we can estimate an average flattening rate of 0.008 dex $\mathrm{kpc}^{-1} \mathrm{Gyr}^{-1}$ during the past $6 \mathrm{Gyr}$. This rate is quite consistent with that from recent $\mathrm{PN}$ data for $[\mathrm{O} / \mathrm{H}]$ (Maciel et al. 2003), and is also in good agreement with the model result of Hou et al. (2000).

\subsection{Disk age-metallicity relation}

The age-metallicity relation (AMR) for the Galactic disk provides useful clues about the chemical evolution history of the Milky Way, and also puts an important constraint on the theoretical models of the disk.

Based on our new open cluster catalogue with many more objects, we found that, statistically, the space distributions of open clusters very likely imply the existence of an age-metallicity relation in the Galactic disk. However, in order to have a more definite conclusion, observational efforts should be added in finding more old clusters.

Acknowledgments. This research was supported by the NSFC (No.19873014, No.10173017, No.10133020) and NKBRSFG 19990754.

\section{References}

Baumgartd, H., Dettbarn, C., \& Wielen, R. 2000, A\&A, 146, 251

Chen, L., Hou, J.L., \& Wang, J.J. 2003, AJ, 125, 1397

Dias, W. S., Lépine, J. R., and Alessi, B. S. 2001, A\&A, 376, 441

Dias, W. S., Alessi, B. S., Moitinho, A., \& Lépine, J. R. 2002, A\&A, 389, 871

Janes, K. A. 1979, ApJS, 39, 135

Hou, J. L., Prantzos, N. \& Boissier, S. 2000, A\&A, 362, 921

Maciel, W. J., Costa, R. D. D. \& Uchida, M. M. M. 2003, A\&A, 397, 667 